\title{
Evaluación de monitores electroquímicos para estudios de corrosión atmosférica del acero carbono
}

\author{
Evaluation of electrochemical monitors for \\ atmospheric corrosion studies of carbon steel
}

Luis Garita-Arce'

Fecha de recepción: 23 de julio del 2013

Fecha de aprobación: 3 de noviembre del 2013

Garita-Arce, L. Evaluación de monitores electroquímicos para estudios de corrosión atmosférica del acero carbono. Tecnología en Marcha. Vol. 27, № 2. Pág 3-13 


\section{Palabras clave}

Corrosión atmosférica; monitores electroquímicos; ruido electroquímico; resistencia de polarización lineal.

\section{Resumen}

El objetivo de la investigación fue evaluar diferentes tipos de monitores electroquímicos para realizar estudios de corrosión atmosférica. Para ello se realizaron corridas en el estado cero de las probetas y condiciones controladas de humedad relativa y temperatura; también se efectuaron corridas a través del tiempo y con probetas activadas hidrofilicamente. Las técnicas electroquímicas utilizadas son la resistencia de polarización líneal (RPL) y el ruido electroquímico (RE), las cuales tienen como variables respuesta la resistencia de polarización (Rp) y la resistencia al ruido $(\mathrm{Rn})$ respectivamente.

Entre las principales conclusiones tenemos que la probeta de tres electrodos idénticos y la de tipo peineta de tres electrodos presentan mejores resultados con respecto a las variaciones de humedad relativa $(H R)$; sin embargo, se recomienda realizar nuevos electrodos de forma de peineta, con distancias menores de separación entre ellos, para así realizar estudios comparándola con la de tres electrodos idénticos.

\section{Key words}

Atmospheric corrosion; electrochemical monitors; electrochemical noise (ECN); linear polarization resistance (LPR).

\begin{abstract}
In the current study, it is the objective to evaluate different types of electrochemical monitors for studies of atmospheric corrosion.

In order to achieve such objective, runs were carried out in the zero state of the specimens, under controlled relativity humidity and temperature. Furthermore, runs were carried out over time and with hydrophilic activated samples. The used electrochemical techniques were: the linear polarization resistance (LPR) and the electrochemical noise $(E C N$,$) which have as response variables the$ polarization resistance (RP) and the noise resistance (RN) respectively. Among the main conclusions, it is that the specimen of three identical electrodes and the comb type one of three electrodes show better results in regards to variations of relative humidity $(\mathrm{RH}$.

However, it is recommended new comb shaped electrodes where the distance between them is less in order to study them by comparing such electrodes with the three identical ones.
\end{abstract}

\section{Introducción}

Existen diferentes métodos para determinar la velocidad de corrosión en los materiales. En lo que respecta a la corrosión atmosférica, el más utilizado es el gravimétrico. Sin embargo, tomando en consideración que todos los factores electroquímicos son significativos en los procesos de corrosión metálica y que operan en la atmósfera, es posible llevar a cabo análisis cinéticos mecanicistas utilizando técnicas electroquímicas. Entre estos métodos están: curvas de polarización, espectroscopía de impedancia, la Resistencia de Polarización Lineal (RPL) y el Ruido electroquímico (RE) (CYTED, 1998)

La técnica del ruido electroquímico se basa en el análisis de registros temporales de potencial y corriente adquiridos sobre un sistema que evo- luciona libremente. Esta técnica se encuentra aún en desarrollo y está siendo utilizada para obtener información sobre la cinética y los mecanismos de los procesos de corrosión. En la actualidad, no existe un consenso sobre los métodos de análisis más adecuados para la interpretación de los datos experimentales (Botana, 2002).

La resistencia de polarización lineal se basa en que las curvas de polarización son prácticamente rectas en un pequeño entorno del potencial de corrosión y su pendiente está relacionada con la velocidad de corrosión. En la práctica se aplica una pequeña polarización y se mide el cambio de corriente para de esa forma con el cociente de esas dimensiones se obtiene la resistencia de polarización (Rp) (González, 1989). 


\section{Monitores Electroquímicos de Corrosión Atmosférica (MECA)}

Para aplicar las técnicas mencionadas se deben construir monitores electroquímicos, los cuales son probetas con pequeñas láminas construidas del metal en estudio que funcionan como electrodos. Las probetas se forman con ayuda de una resina resistente al ataque químico y a cualquier medio; en ellas las láminas de metal quedan lo suficientemente cerca como para que una pequeña capa de humedad (humedad relativa baja) del medio sirva como electrolítico.

La humedad relativa juega un papel importante en la determinación de la agresividad atmosférica ya que, junto a la temperatura, se determina el tiempo de humectación, que es un factor importante en la categorización de la agresividad de la atmósfera, según la Norma ISO 9223 (Corrosion of metals and alloys- Corrosivity of atmospheres-Classification).

Dado lo anterior, existen diferentes tipos de MECA de acuerdo a su forma geométrica y la cantidad de electrodos para que puedan funcionar con capas de electrolito delgado.

\section{Probeta de 3 electrodos idénticos}

Esta probeta consiste en un arreglo de 3 electrodos idénticos (ver figura I) colocados lo más cerca posible entre ellos dentro de la resina, para así lograr que la humedad del aire funcione como electrolito a pesar de ser una capa delgada.

Probeta tipo peineta de dos electrodos

La probeta con forma de peineta consiste en un arreglo de los electrodos con la forma geométrica de un peine (ver figura 2) y el objetivo principal de esta configuración es aumentar el área entre los electrodos. Utilizando el arreglo tipo peineta se puede aumentar el área de contacto entre los electrodos y el electrolito manteniéndolos lo más cerca posible.

El esquema de medición de este sistema se basa en alternar la medida de corriente y de potencial sobre el par de electrodos de trabajo (Botana, 2002).

Probeta tipo peineta de tres electrodos

De igual forma que los electrodos convencionales, el arreglo peineta se puede trabajar utilizando 3 electrodos (ver figura 3). Esta probeta consta de una lámina central aislada que no recibe polarización durante las medidas, es decir, actúa como electrodo de referencia, mientras los electrodos dentados funcionan como electrodo de trabajo y contraelectrodo (Gonzáles, Escudero, Lopez, Simancas, \& Morcillo, 2004).

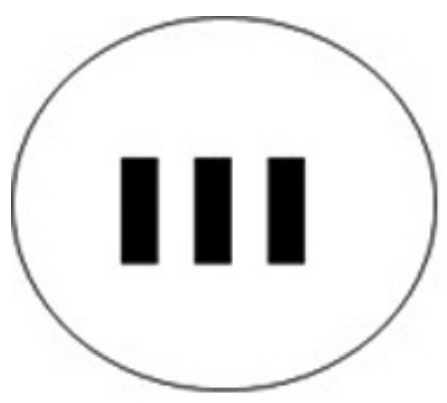

Figura I. Monitor electroquímico de tres electrodos idénticos. Fuente: Autor.

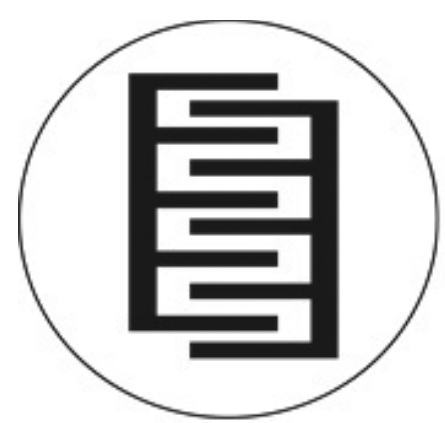

Figura 2. Monitor electroquímico de peineta de dos electrodos. Fuente: Autor.

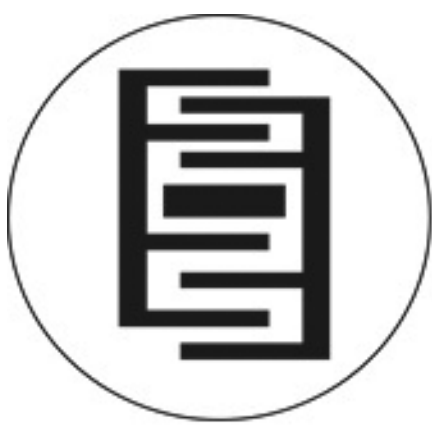

Figura 3. Monitor electroquímico de peineta de tres electrodos. Fuente: El autor. 


\section{Carácter hidrofílico de la superficie}

Se debe tener en cuenta que independientemente de que la superficie metálica aparezca mojada por efecto de la lluvia, o bien se produzca un aumento de la HR y la consiguiente condensación, se dan varios mecanismos que permiten la formación de la película húmeda. Por otra parte, a medida que crece este espesor de película, la velocidad de corrosión aumenta hasta alcanzar un máximo para espesores del orden de los I00 $\mu$ m (Otero Huerta, 200 I).

En algunos casos se realiza una activación iónica a la superficie de la probeta mediante $\mathrm{NaOH}$, con el objetivo que adquiera ese carácter hidrofilico, esto para los casos en donde la humedad relativa se encuentre por debajo de la crítica y la capa de electrolito sea muy delgada (Tres, 200 I).

\section{Metodología}

Para preparar las distintas probetas, los electrodos se construyeron a partir de un acero carbono estructural el cual cumple la composición química según norma ASTM A36/A36M-12 Standard Specification for Carbon Structural Steel de acuerdo a los resultados del cuadro I. La determinación de la composición química se realiza de acuerdo con la norma ASTM E4I5-08 Standard Test Method for Atomic Emission Vacuum Spectrometric Analysis of Carbon and Low-Alloy Steel. Dichos electrodos son embebidos en una resina dejando una cara de estos expuesta al ambiente. La resina es un material aislante resistente a cualquier ataque químico de

Cuadro I. Composición química del material utilizado en la construcción de los monitores electroquímicos.

\begin{tabular}{|c|c|}
\hline Elementos & Composición $(\% \mathrm{~m} / \mathrm{m})$ \\
\hline Carbono & 0,239 \\
\hline Manganeso & 0,749 \\
\hline Fósforo & 0,010 \\
\hline Azufre & 0,029 \\
\hline Silicio & 0,274 \\
\hline Cobre & 0,031 \\
\hline
\end{tabular}

la atmósfera. Para el caso de las probetas activadas, estas se dejaron en una disolución de 0,I M de $\mathrm{NaOH}$ a una temperatura de $80^{\circ} \mathrm{C}$ durante dos horas.

Cabe recalcar que en las pruebas en estado cero y en las que se corrieron a través del tiempo se usaron probetas cuya superficie no fue activada. La pruebas de electroquímica de resistencia de polarización línea y ruido electroquímico se realizan de acuerdo a normas ASTM G 59-97 Standard Test Method for Conducting Potentiodynamic Polarization Resistance Measurements y ASTM G-199-09 Standard Guide for Electrochemical Noise Measurements respectivamante.

\section{Pruebas en estado cero}

Las pruebas se realizan en un cuarto con atmósfera controlada, donde la temperatura promedio es de $25^{\circ} \mathrm{C}$ aproximadamente y la humedad relativa del $60 \%$. A las muestras se les determina la resistencia al ruido electroquímico (Rn). Después de cada corrida, la probeta será lijada, enjuagada con agua destilada y se dejará en reposo durante un período de una hora, para volver a realizar la prueba de ruido nuevamente. Durante todo el experimento se trabaja con las mismas 3 muestras iniciales. Se realiza un total de 10 corridas para cada probeta.

Pruebas durante el tiempo

Una vez finalizadas las 10 corridas, las muestras son lijadas nuevamente y se dejan en una atmósfera natural con una tasa de depositación de contaminantes de $\mathrm{SO}_{2}$ de $6,56 \mathrm{mg} / \mathrm{m}^{2} /$ día y para el $\mathrm{Cl}^{-}$de $12,02 \mathrm{mg} / \mathrm{m}^{2} / \mathrm{d}$ á y un tiempo de humectación expresado fraccionalmente de 0,56 donde, según estos datos y de acuerdo a la norma ISO 92232012, se clasifica como C3, es decir, atmósfera de agresividad corrosiva media. Se realizan nuevas pruebas de ruido electroquímico y de resistencia de polarización lineal, anotando el tiempo entre cada medida. Se deben anotar las mediciones de temperatura y humedad relativa cada vez que se vaya a realizar una corrida, ya que el objetivo principal es observar los cambios en las probetas con el tiempo y los cambios de la atmósfera.

\section{Probetas con activación de la superficie}

En esta parte el objetivo es determinar si la activación de la superficie en la probeta aporta una mejora a los resultados. Por ende, se vuelven a 
formar las probetas de 3 electrodos y las de peineta de 3 electrodos. Se confecciona un par de cada probeta y se expone una probeta de 3 electrodos y una de peineta a una disolución de 0,I M de $\mathrm{NaOH}$ a una temperatura de $80^{\circ} \mathrm{C}$ durante dos horas. Una vez listas, las probetas son expuestas a la atmósfera natural antes mencionada y se observa su comportamiento en el tiempo, por medio de las técnicas de RE y RPL, para observar y comparar el comportamiento de las probetas que fueron activadas con la disolución de $\mathrm{NaOH}$ y las que no fueron activadas. Se tendrá una probeta de cada tipo, activadas y no activadas.

\section{Discusión de resultados}

Pruebas en estado cero

Para la primera parte del experimento, donde las muestras se encuentran todas en estado cero de exposición bajo condiciones ambientales controladas, se realiza el análisis de varianza respectivo entre los valores de Rn obtenidos por cada probeta utilizada (ver en el cuadro 2 los valores de Rn). Al observar el resultado del análisis (ver cuadro 3) se concluye que estadísticamente algún tratamiento se desvía de la media de los otros dos tratamientos, por lo que se concluye que no hay igualdad entre los $\mathrm{Rn}$ obtenidos en el laboratorio.

Dado que el análisis de varianza solamente establece que algún tratamiento puede ser diferente, se recurre a una prueba de Tukey (ver cuadro 4), tras lo cual se puede afirmar que los resultados de las Rn calculadas con la probeta en forma de peineta de 3 electrodos son los valores significativos que se salen del límite de significancia. Por lo tanto, por medio de esta prueba podemos afirmar con un 95\% de probabilidad que al utilizar la probeta en forma de peineta de 3 electrodos se producen resultados diferentes a las demás. Por otro lado, también se puede afirmar que no hay diferencia significativa entre los resultados obtenidos con la probeta de 3 electrodos y la de peineta de 2 electrodos.

Pruebas a través del tiempo

Se realizan las pruebas de RE y RPL a las probetas de tres electrodos, mientras que a la probeta de dos electrodos solamente se le aplica RE.

El tiempo total de exposición de las muestras a la atmósfera es cercano a las 500 horas, durante las cuales estuvieron expuestas a diversos cambios de temperatura, humedad, viento, lluvia, según el estado meteorológico.

Cuadro 2.Valores de la resistencia al ruido (Rn) para las probetas en estado cero bajo atmósfera controlada.

\begin{tabular}{|c|c|c|c|c|c|}
\hline Corrida & $T\left({ }^{\circ} \mathrm{C}\right)$ & HR \% & $\begin{array}{c}\text { Rn Probeta } \\
\begin{array}{c}\text { electrodos } \\
\left(\mathrm{Ohm} * \mathrm{~cm}^{2}\right)\end{array}\end{array}$ & $\begin{array}{c}\text { Rn peineta 3 electrodos } \\
\left(\mathrm{Ohm} \mathrm{cm}^{2}\right)\end{array}$ & $\begin{array}{c}\text { Rn Peineta 2 electrodos } \\
\left(\mathrm{Ohm}^{*} \mathrm{~cm}^{2}\right)\end{array}$ \\
\hline 1 & 24,9 & 66 & $2,27 \mathrm{E}+07$ & $5,72 \mathrm{E}+07$ & $1,65 \mathrm{E}+06$ \\
2 & 25 & 66 & $6,09 \mathrm{E}+06$ & $7,64 \mathrm{E}+07$ & $3,88 \mathrm{E}+06$ \\
3 & 24,7 & 59 & $3,65 \mathrm{E}+06$ & $6,49 \mathrm{E}+07$ & $4,01 \mathrm{E}+06$ \\
4 & 23,8 & 59 & $8,85 \mathrm{E}+07$ & $9,22 \mathrm{E}+07$ & $4,19 \mathrm{E}+06$ \\
5 & 25,7 & 51 & $7,11 \mathrm{E}+06$ & $5,07 \mathrm{E}+07$ & $1,67 \mathrm{E}+06$ \\
6 & 27,2 & 55 & $8,36 \mathrm{E}+06$ & $7,42 \mathrm{E}+07$ & $2,26 \mathrm{E}+06$ \\
7 & 26 & 63 & $2,83 \mathrm{E}+07$ & $7,77 \mathrm{E}+07$ & $2,08 \mathrm{E}+06$ \\
8 & 25,7 & 61 & $3,79 \mathrm{E}+06$ & $7,00 \mathrm{E}+07$ & $2,51 \mathrm{E}+06$ \\
9 & 25,4 & 61 & $5,20 \mathrm{E}+06$ & $1,11 \mathrm{E}+08$ & $3,16 \mathrm{E}+06$ \\
10 & 25 & 69 & $1,61 \mathrm{E}+08$ & $6,97 \mathrm{E}+07$ & $4,18 \mathrm{E}+06$ \\
\hline
\end{tabular}


Cuadro 3: Resultado del ANOVA de las pruebas en estado cero con atmósfera controlada para la Rn.

\begin{tabular}{|c|c|c|c|c|c|c|}
\hline $\begin{array}{c}\text { Origen de las } \\
\text { variaciones }\end{array}$ & $\begin{array}{c}\text { Suma de } \\
\text { cuadrados }\end{array}$ & $\begin{array}{c}\text { Grados de } \\
\text { libertad }\end{array}$ & $\begin{array}{c}\text { Promedio de los } \\
\text { cuadrados }\end{array}$ & F & Probabilidad & $\begin{array}{c}\text { Valor crítico } \\
\text { para F }\end{array}$ \\
\hline Entre grupos & $2,57 \mathrm{E}+16$ & 2 & $1,28 \mathrm{E}+16$ & 12,943 & 0,0001 & 3,35 \\
\hline $\begin{array}{c}\text { Dentro de los } \\
\text { grupos }\end{array}$ & $2,68 \mathrm{E}+16$ & 27 & $9,93 \mathrm{E}+14$ & & & \\
\hline Total & $5,25 \mathrm{E}+16$ & 29 & & & & \\
\hline
\end{tabular}

Cuadro 4. Resultados del análisis de la prueba de Tuckey para la Rn de las pruebas en estado cero.

\begin{tabular}{|c|c|c|c|c|c|}
\hline Contraste & Diferencia & $\begin{array}{c}\text { Diferencia } \\
\text { estandarizada }\end{array}$ & $\begin{array}{c}\text { Valor } \\
\text { crítico }\end{array}$ & Pr $>$ Dif & Significativo \\
\hline $\begin{array}{c}\text { Peineta 3 electrodos vs Peineta 2 } \\
\text { electrodos }\end{array}$ & 71457878,16 & 5,07 & 2,48 & $<0,0001$ & $\mathrm{Si}$ \\
\hline $\begin{array}{c}\text { Peineta 3 electrodos vs Probeta 3 } \\
\text { electrodos idénticos }\end{array}$ & 40919210,00 & 2,90 & 2,48 & 0,019 & $\mathrm{Si}$ \\
\hline $\begin{array}{c}\text { Probeta 3 electrodos idénticos vs } \\
\text { Peineta 2 electrodos }\end{array}$ & 30538668,16 & 2,17 & 2,48 & 0,095 & $\mathrm{No}$ \\
\hline
\end{tabular}

Al comparar los resultados obtenidos de Rn mediante la técnica de RE entre las 3 probetas utilizadas, se observa que durante el tiempo de exposición las probetas mantienen comportamientos diferentes entre sí (ver figura 4). Sin embargo, es notorio que la probeta de 3 electrodos idénticos y la probeta de 3 electrodos tipo peineta presentan comportamiento más cercanos a través de todo el tiempo.
A la hora de realizar el mismo gráfico de comportamiento de los resultados de Rp obtenidos por el método de RPL, se puede afirmar que la probeta de 3 electrodos idénticos y la de 3 electrodos tipo peineta tienen comportamientos similares, es decir, la curva crece y decrece en la mismas unidades de tiempo. Ambas llegan a un valor mínimo en común cercano a las 300 horas, con un valor aproximado

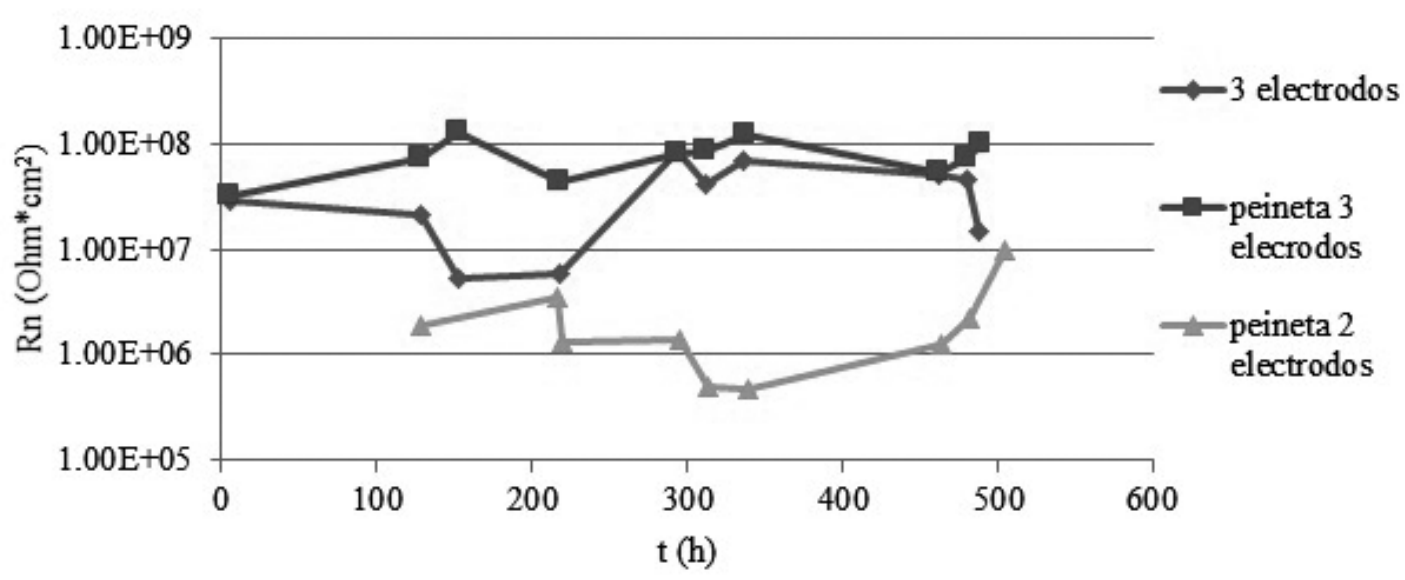

Figura 4. Comportamiento de la Rn para las tres probetas durante el tiempo.Valores obtenidos por el método de ruido electroquímico. Fuente: Autor. 


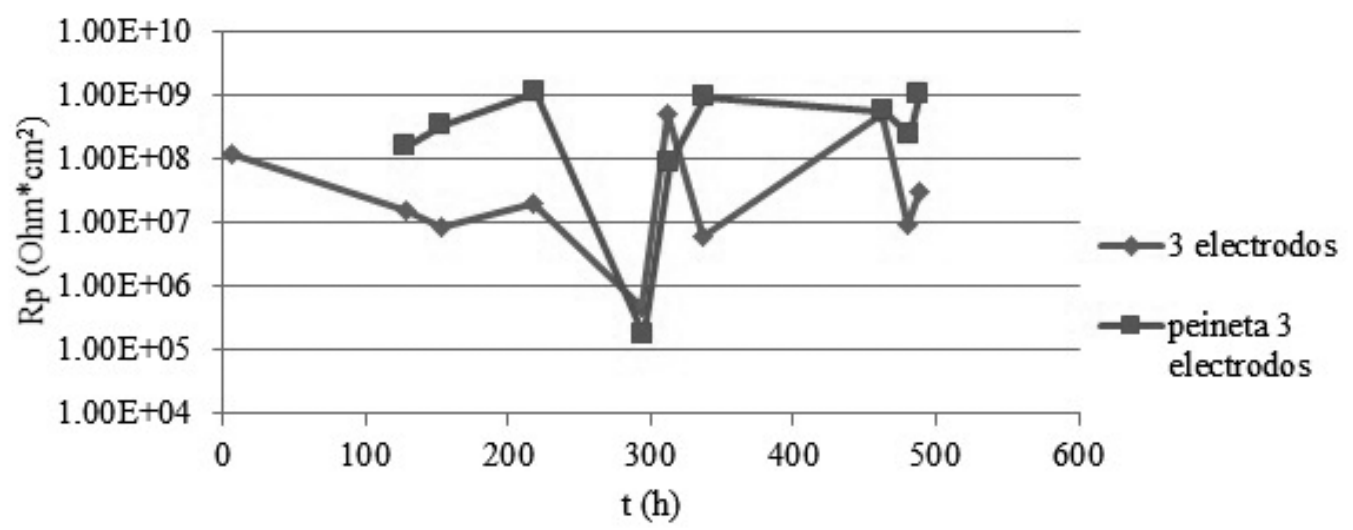

Figura 5. Comportamiento de Rp para las probetas durante el tiempo.Valores obtenidos por el método de LPR. Fuente: Autor.

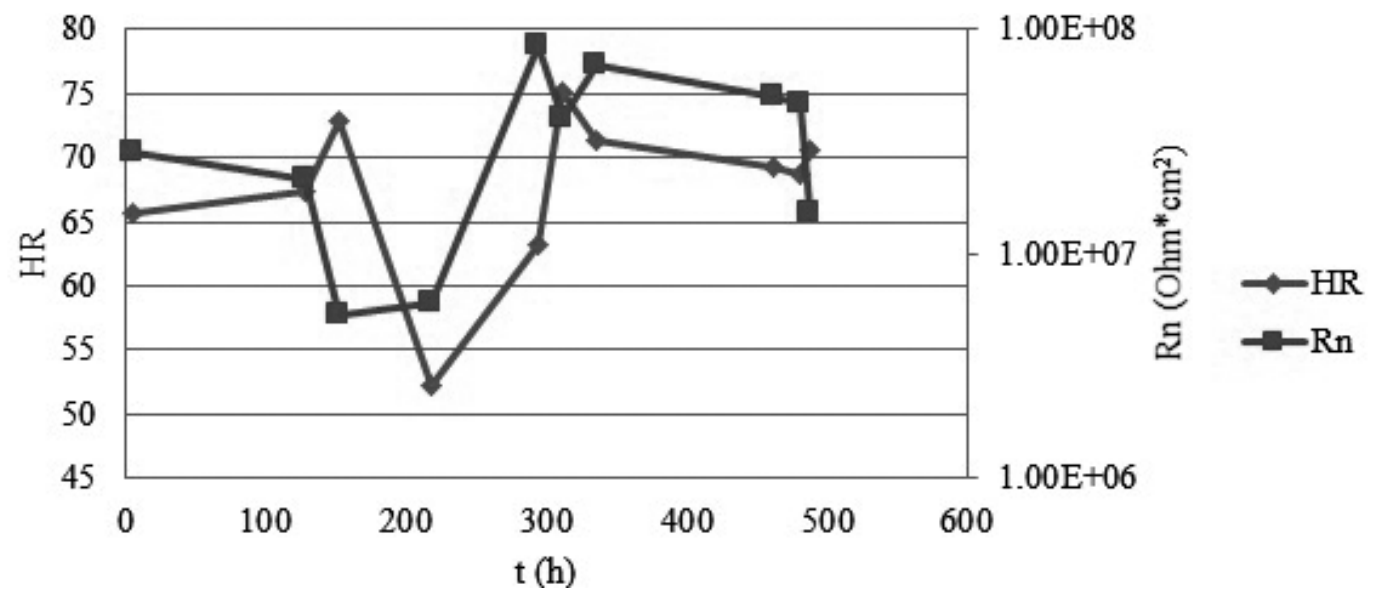

Figura 6. Relación de la resistencia al ruido y la humedad con las pruebas en el tiempo en la probeta de 3 electrodos idénticos. Fuente: Autor.

de $3,90 \times 10^{5} \mathrm{Ohm}^{*} \mathrm{~cm}^{2}$. Se pueden observar los resultados en la figura 5 .

Se debe tener en cuenta para los análisis siguientes que a medida que el espesor de la película crece, la velocidad de corrosión aumenta, hasta alcanzar un máximo para espesores del orden de los 100 um y que los espesores a partir de I mm son considerados como condiciones de inmersión. Por otra parte, los espesores de humedad visible se encuentran a partir de las 10 mm (Otero Huerta, 200I).

Teniendo lo anterior en cuenta, se debe indicar que siempre se trabajó con películas invisibles y humedad relativa menor a 100\%. Ahora bien, al aumentar el valor de la humedad relativa, aumenta la facilidad de formarse la película de electrolito sobre la probeta, lo cual hace que disminuyan tanto la $\mathrm{Rn}$ como la Rp. Según los resultados, la probeta de 3 electrodos idénticos es la que presenta los resultados más cercanos a lo esperado.

En los resultados de la probeta de 3 electrodos, durante las primeras 200 horas de exposición se observa el comportamiento inversamente proporcional de la humedad con la resistencia. Los resultados se pueden observar en la figura 6 .

Como se mencionó anteriormente, los resultados de las demás probetas no se ajustan al comportamiento esperado, en lo que respecta a la humedad relativa. En la probeta de peineta de 3 electrodos se da el comportamiento esperado de la humedad con el Rn en el intervalo de las 300 horas a las 340 
horas aproximadamente, mientras que en la probeta de peineta de 2 electrodos se observa ese mismo comportamiento en el intervalo de las 463 horas a las 481 horas de exposición aproximadamente. Se pueden observar los resultados en las figuras 7 y 8 .

El comportamiento de las resistencias a la polarización con la humedad relativa en la probeta de 3 electrodos idénticos se da como se tenía esperado en la gran mayoría del tiempo de exposición. En este caso se observa como disminuye la Rp conforme aumenta la humedad relativa y viceversa después de 100 horas de exposición (figura 9).

Los resultados del comportamiento de la Rp con la humedad en la probeta de peineta de 3 electrodos (ver figura I0) también muestran el comportamiento esperado, después de aproximadamente 150 horas de exposición.

En síntesis, se podría decir que el comportamiento esperado de las resistencias con respecto a la humedad relativa se da en las probetas de 3 electrodos, mientras que la de 2 electrodos no presenta similitudes con respecto a las dos anteriores.

Monitores electroquímicos con activación hidrofílica en su superficie

El tiempo de exposición de los electrodos activados y no activados es de un total de 654 horas aproximadamente. Al inicio de las pruebas de las probetas activadas, solamente la probeta de 3 electrodos idénticos (ver figura ll) presenta el

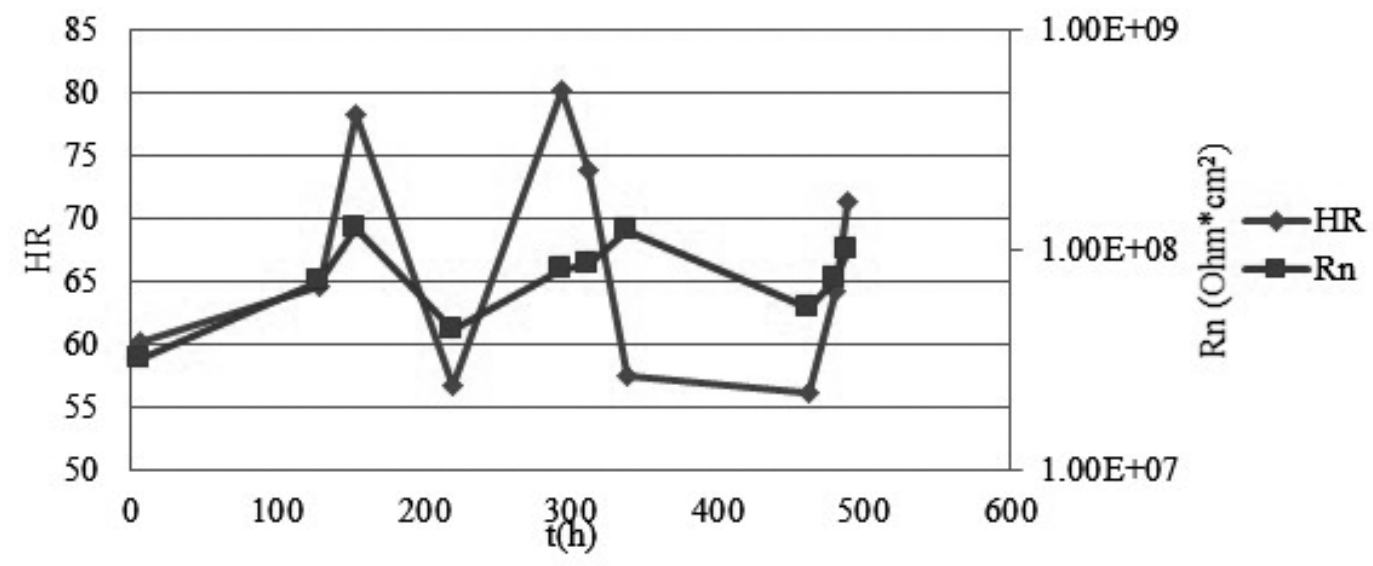

Figura 7. Comportamiento del $\mathrm{Rn}$ con respecto a la humedad durante el tiempo en la probeta de peineta de 3 electrodos. Fuente: Autor.

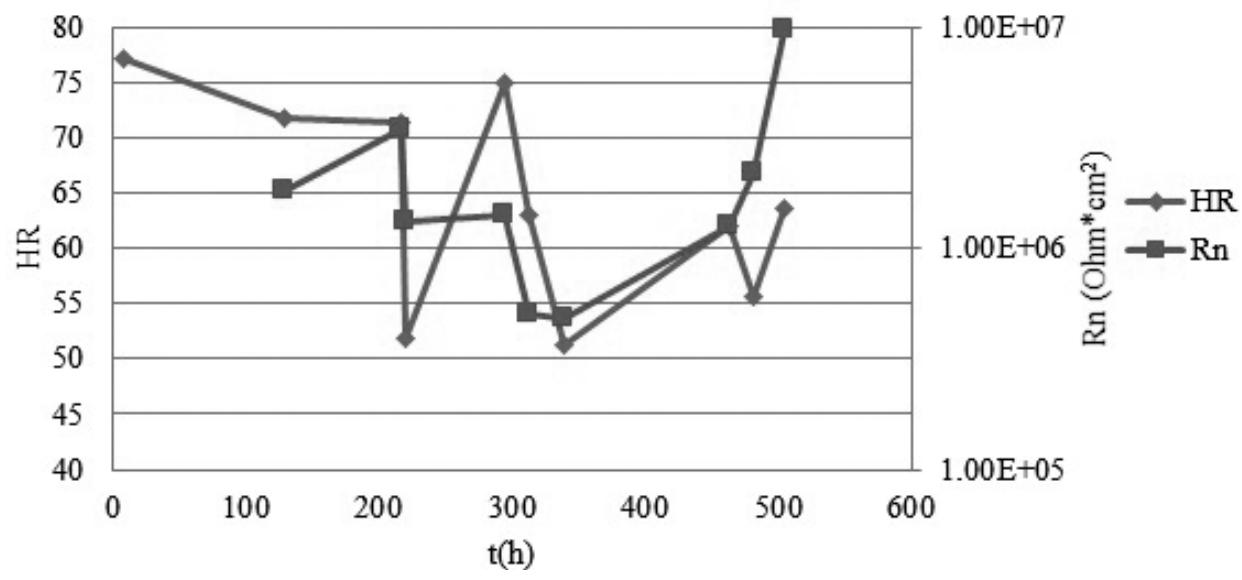

Figura 8. Comportamiento del Rn con la humedad durante el tiempo en la probeta de peineta de 2 electrodos. Fuente: Autor. 


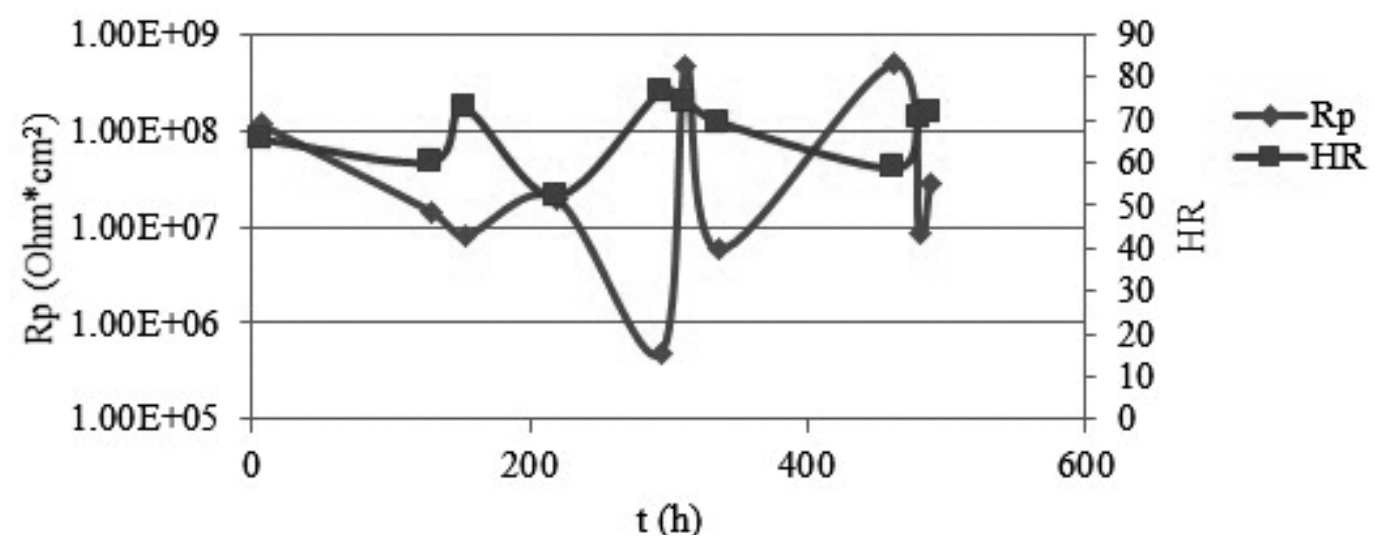

Figura 9. Comportamiento de la Rp con la humedad relativa durante el tiempo en la probeta de 3 electrodos. Fuente: Autor.

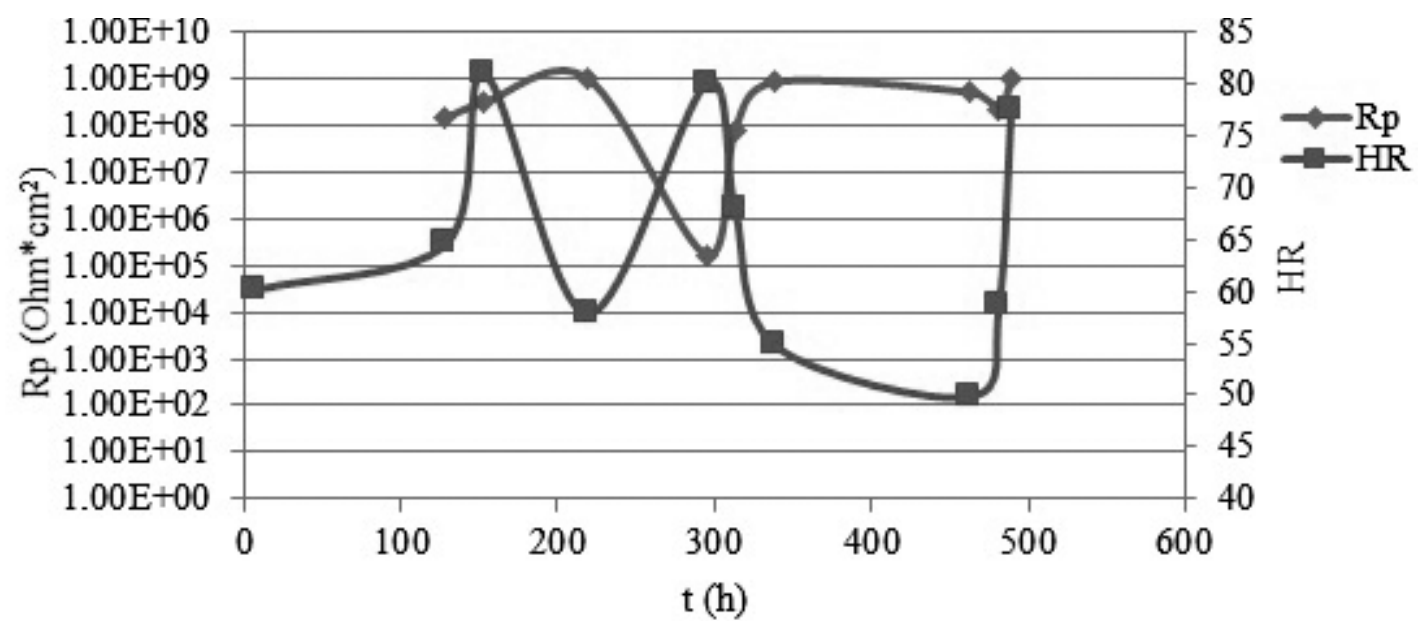

Figura 10. Comportamiento de la Rp con la humedad durante el tiempo en la probeta de peineta de 3 electrodos. Fuente: Autor.

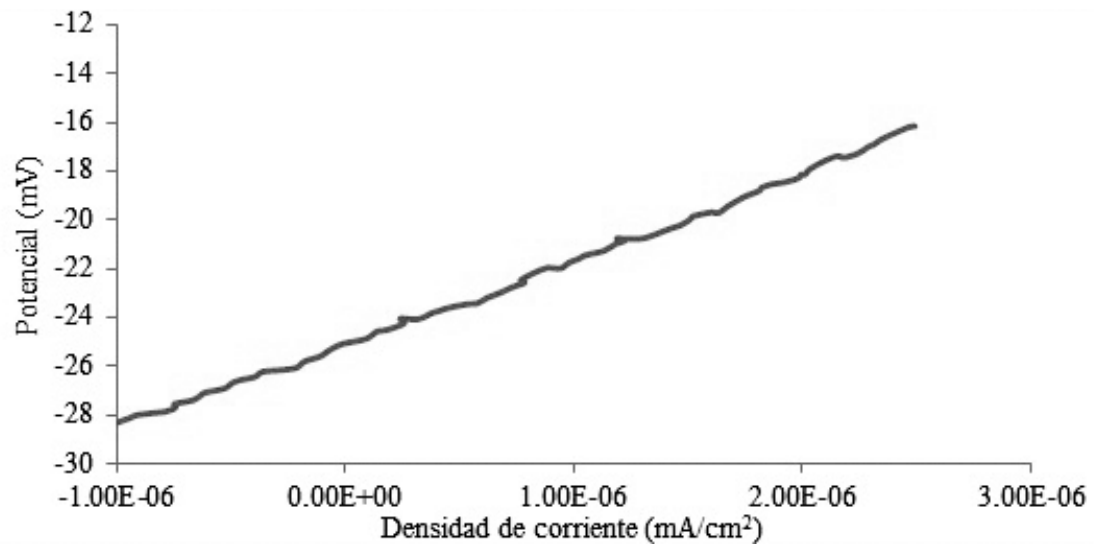

Figura | I. Primer corrida de la curva de resistencia de polarización lineal a $T=26.7 H R=57,4 \%$. Probeta activada de tres electrodos idénticos. Fuente: Autor. 


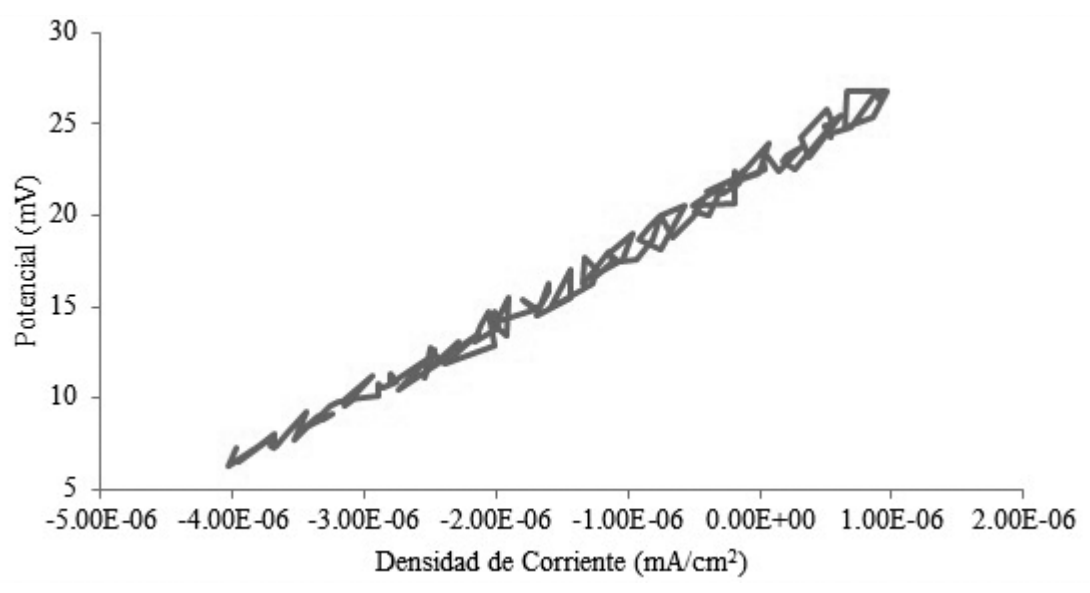

Figura 12. Primer corrida de la curva de resistencia de polarización lineal a $T=26.7 H R=57,4 \%$. Probeta activada de tres electrodos tipo peineta. Fuente: Autor.

comportamiento idóneo de una curva de LPR, a pesar de tener una humedad relativa del 57,4\%; mientras que la de tipo peineta no presentó ese comportamiento (ver figura 12). Un factor importante que puede explicar esta diferencia es la distancia entre los electrodos, ya que en la probeta de 3 electrodos idénticos, la distancia entre ellos es menor a $1 \mathrm{~mm}$, comparada con la distancia entre los electrodos en forma de peineta que es de aproximadamente $3 \mathrm{~mm}$. Aproximadamente entre las 174 y las 200 horas de exposición, la resina se desactiva y pierde su carácter hidrofilico y los resultados son similares a probetas no activadas.

\section{Conclusiones}

En condiciones controladas, en el estado cero de las probetas y mediante la prueba de Tukey, se concluye que la probeta con forma de peineta de 3 electrodos es la que presenta diferencias significativas con respecto a las demás probetas, con un 95\% de confianza.

En la prueba a través del tiempo, cuando se exponen los valores de resistencia de RPL y de RE con respecto a la humedad relativa; las probetas muestran un comportamiento típico de acuerdo a las variaciones de humedad, con excepción de la probeta de 2 electrodos.

De acuerdo a las gráficas de RPL, la probeta activada que presenta un mejor comportamiento es la de
3 electrodos idénticos, sin embargo, comienza a perder su carácter hidrofilico al transcurrir las 170 horas aproximadamente. Después de este tiempo, las gráficas de LPR pierden su comportamiento típico.

Se recomienda realizar nuevos electrodos de forma de peineta en los cuales las distancias de separación entre ellos sea menor, para así realizar estudios comparándola con la de tres electrodos idénticos; además de probar otras probetas de diferentes geometrías, como las circulares.

Se recomienda estudiar más a fondo el tiempo en que disminuye el carácter hidrofilico de la superficie de la probeta.

\section{Bibliografía}

ASTM (1997). Standard test method for conducting potentiodynamic polarization resistance measurements. Estados Unidos: ASTM.

ASTM (2008). Standard Test Method for Atomic Emission Vacuum Spectrometric Analysis of Carbon and Low-Alloy Steel. Estados Unidos: ASTM.

ASTM. (2009). Standard guide for electrochemical noise measurement. Estados Unidos: ASTM.

ASTM. (20I2). Standard Specification for Carbon Structural steel. United States: ASTM.

Botana, J. (2002). Ruido electroquímico. Métodos de análisis. España: SEPTEM.

CYTED. (1998). Corrosión y protección de metales en las atmosféras de Iberoamérica. España: Gráficas Salue S.A. 
Gonzáles, J., Escudero, E., López, V., Simancas, J. \& Morcillo, M. (2004). Durabilidad del aluminio desnudo y anodizado en atmósferas de muy diferentes corrosividades. Revista de Metalurgia 259-261.

González, J. (1989). Control de la corrosión: estudio y medida por técnicas electroquímicas. Madrid: GRAFIPREN S.A.

International Organization for Standardization. (20 I 2). ISO 9223 Corrosion of Metal and Alloys- Corrosivity of atmospheres- classification. Suiza: ISO.
Otero Huerta, E. (200 I). Corrosión y degradación de los materiales. Madrid: Síntesis SA.

Sánchez, J., Bethencourt, M., González, L. \& Botana, E. (2009). Medida de ruido electroquímico para el estudio de procesos de corrosión de aleaciones metálicas. Revista de Metalurgia 143-156.

Tres, G. (200I). Monitoreo por Ruido Electroquímico de la Corrosión del Acero en una Atmósfera Contaminada. Revista Internacional de Contaminación Ambiental 17|- 778. 